\title{
CODIMENSION ONE ISOMETRIC IMMERSIONS BETWEEN LORENTZ SPACES

\author{
BY
}

L. K. GRAVES

In memory of my father, Lucius Kingman Graves

\begin{abstract}
The theorem of Hartman and Nirenberg classifies codimension one isometric immersions between Euclidean spaces as cylinders over plane curves. Corresponding results are given here for Lorentz spaces, which are Euclidean spaces with one negative-definite direction (also known as Minkowski spaces). The pivotal result involves the completeness of the relative nullity foliation of such an immersion. When this foliation carries a nondegenerate metric, results analogous to the Hartman-Nirenberg theorem obtain. Otherwise, a new description, based on particular surfaces in the three-dimensional Lorentz space, is required.
\end{abstract}

The theorem of Hartman and Nirenberg [HN] says that, up to a proper motion of $\mathbf{E}^{n+1}$, all isometric immersions of $\mathbf{E}^{n}$ into $\mathbf{E}^{n+1}$ have the form

$$
\text { id } \times c: \mathbf{E}^{n-1} \times \mathbf{E}^{1} \rightarrow \mathbf{E}^{n-1} \times \mathbf{E}^{2}
$$

where $c: \mathbf{E}^{1} \rightarrow \mathbf{E}^{2}$ is a unit speed plane curve and the factors in the product are orthogonal. A proof of the Hartman-Nirenberg result also appears in $\left[\mathbf{N}_{\mathbf{1}}\right]$. The major step in proving the theorem is showing that the relative nullity foliation, which is spanned by those tangent directions in which a local unit normal field is parallel, has complete leaves. These leaves yield the $\mathbf{E}^{n-1}$ factors in $(0.1)$. The one-dimensional complement of the relative nullity foliation gives rise to the curve $c$.

This paper studies isometric immersions of $\mathbf{L}^{n}$ into $\mathbf{L}^{n+1}$, where $\mathbf{L}^{n}$ denotes the $n$-dimensional Lorentz (or Minkowski) space. Its chief goal is the classification, up to a proper motion of $\mathbf{L}^{n+1}$, of all such immersions.

Part I introduces the terminology and states the elementary results required to achieve the desired classification. In particular, null curves-curves in $\mathbf{L}^{n}$ whose tangent vectors, although nonzero, have zero "length"-and appropriate frames for them are discussed, the theory of Lorentz hypersurfaces in $\mathbf{L}^{n+1}$ is described, and examples are exhibited.

Received by the editors February 22, 1977 and, in revised form, August 6, 1978.

AMS (MOS) subject classifications (1970). Primary 53C50, 53C40; Secondary 83A05.

Key words and phrases. Lorentz spaces, isometric immersions, null curves, null frames, relative nullity foliation, (non)degenerate relative nullities, complete relative nullities, Hartman-Nirenberg theorem, cylinders over plane curves, $B$-scrolls. 
The major task of demonstrating the completeness of the leaves of the relative nullity foliation is accomplished in Part II. The case where the relative nullity spaces inherit nondegenerate metrics can be handled virtually in the same manner as the Euclidean case. However, when the relative nullities are "degenerate" (defined in \$6), different techniques are required. It is shown in passing that the cases are mutually exclusive in nontrivial cases.

Part III contains the classification results. When the relative nullity spaces are nondegenerate, the immersion has one of the following forms:

$$
\begin{aligned}
& \text { id } \times c: \mathbf{E}^{n-1} \times \mathbf{L}^{1} \rightarrow \mathbf{E}^{n-1} \times \mathbf{L}^{2}, \\
& \text { id } \times c: \mathbf{L}^{n-1} \times \mathbf{E}^{1} \rightarrow \mathbf{L}^{n-1} \times \mathbf{E}^{2} .
\end{aligned}
$$

In (0.2) and (0.3), $c$ is unit-speed in the sense that $\langle d c / d s, d c / d s\rangle$ is -1 and +1 , respectively. Two theorems cover the case when the relative nullities are degenerate. Theorem (9.7) characterizes the class of isometric immersions of $\mathbf{L}^{2}$ into $\mathbf{L}^{3}$ with this property as the class of $B$-scroll immersions, which are described by Example (3.9) and Theorem (3.13). Then the general isometric immersion $\mathbf{L}^{n} \rightarrow \mathbf{L}^{n+1}$ with degenerate relative nullity, according to Theorem (9.8), has the form

$$
\text { id } \times g: \mathbf{E}^{n-2} \times \mathbf{L}^{2} \rightarrow \mathbf{E}^{n-2} \times \mathbf{L}^{3}
$$

where $g$ is a $B$-scroll immersion.

Most of the results in this paper are taken from the author's Brown University doctoral dissertation. The author gratefully acknowledges the generous encouragement and advice given him by Professor Katsumi Nomizu.

\section{PART I. ElEMENTARY LORENTZ GEOMETRY}

1. Remarks on $\mathbf{L}^{n}$ and the Lorentz group. Consider the real $\boldsymbol{n}$-dimensional vector space $\mathbf{R}^{n}$ with the standard basis $\left\{e_{0}, e_{1}, \ldots, e_{n-1}\right\}$. Let $\langle$,$\rangle denote$ the (indefinite) inner product on $\mathbf{R}^{n}$ whose matrix with respect to the standard basis is

$$
S=\left[\begin{array}{c|ccc}
-1 & & & \\
\hline & 1 & & \\
& & \ddots & \\
& & & 1
\end{array}\right] .
$$

Then $\langle$,$\rangle is called the Lorentz metric on \mathbf{R}^{n} ; \mathbf{R}^{n}$ together with this metric is called the $n$-dimensional Lorentz space and is denoted by $\mathrm{L}^{n}$. A vector $X$ in $\mathrm{L}^{n}$ is called time-like or space-like according to whether $\langle X, X\rangle$ is negative or positive. If $|\langle X, X\rangle|=1$, then $X$ is called a unit vector of its type. If 
$\langle X, X\rangle=0$, then $X$ is called a light-like or null vector. Time- and light-like vectors are causal vectors.

The proof of the following result is standard.

TheOREM (1.1). Let $P$ be a $k$-dimensional subspace of $\mathbf{L}^{n}$. Then exactly one of the following statements about $P$ is true:

(i) $P=\mathbf{L}^{k}$, and $\langle\rangle \mid$,$p is nondegenerate;$

(ii) $P=\mathbf{E}^{k}$, and $\langle\rangle \mid$,$p is nondegenerate;$

(iii) $\langle\rangle \mid$,$p is degenerate, and in this case (and only in this case)$

$$
P=\mathbf{E}^{k-1} \oplus \operatorname{Span}\{\xi\}
$$

where $\langle\xi, \xi\rangle=0$ and $\xi$ is orthogonal to $\mathbf{E}^{k-1}$.

Define $\mathrm{O}(1, n-1)$ to be the subgroup of $\mathrm{GL}(n, \mathbf{R})$ consisting of those transformations which preserve the Lorentz metric. In terms of matrices, we may write

$$
\mathrm{O}(1, n-1)=\left\{U \in \mathrm{GL}(n, \mathbf{R}): U S U^{T}=S\right\}
$$

where $U^{T}$ denotes the transpose of $U . \mathrm{O}(1, n-1)$ is the group of Lorentz transformations on $\mathbf{R}^{n}$. An ordered basis for $\mathbf{L}^{n}$ is a frame; $\left(e_{0}, \ldots, e_{n-1}\right)$ is the standard (orthonormal) frame. An orthonormal frame for $\mathbf{L}^{n}$ is the image of the standard frame under a Lorentz transformation.

The subgroup $\mathrm{SO}^{+}(1, n-1)$ of $\mathrm{O}(1, n-1)$ whose elements $U$ satisfy $\operatorname{det} U=1$ and $\left\langle U e_{0}, e_{0}\right\rangle \leqslant-1$ is the component of the identity in $\mathrm{O}(1, n-$ 1) and is called the group of proper Lorentz transformations.

Define $o(1, n-1)$ to be the set of $n \times n$ matrices $Y$ satisfying $S Y^{T} S=$ $-Y$. It is the Lie algebra of $\mathrm{SO}^{+}(1, n-1)$, and each element of it has the form

$$
\left[\begin{array}{c|c}
0 & X^{T} \\
\hline X & K
\end{array}\right]
$$

where $X \in \mathbf{R}^{n-1}$ and $K$ is an $(n-1) \times(n-1)$ skew-symmetric matrix.

LeMma (1.4). Let $s \mapsto U(s)$ be a curve in $\mathrm{O}(1, n-1)$. Then

$$
\frac{d U}{d s} S U^{T}+U S \frac{d U^{T}}{d s}=0
$$

Proposition (1.5). Let $s \mapsto U(s)$ be a curve in $\mathrm{GL}(n, \mathbf{R})$. Then $U(s)$ lies in $\mathrm{O}(1, n-1)$ if and only if $U\left(s_{0}\right) \in \mathrm{O}(1, n-1)$ for some $s_{0} \in \mathbf{R}$ and $U^{-1} d U / d s \in o(1, n-1)$. 
2. Null curves and frames in $\mathbf{L}^{3}$. In this section, the ambient space for all curves, frames, etc., is $\mathbf{L}^{3}$.

A null frame is an ordered triple of vectors (or, from another point of view, a matrix):

$$
F=(A, B, C)=\left[\begin{array}{lll}
a_{0} & b_{0} & c_{0} \\
a_{1} & b_{1} & c_{1} \\
a_{2} & b_{2} & c_{2}
\end{array}\right]
$$

where $A$ and $B$ are null vectors satisfying $\langle A, B\rangle=-1, C$ is a unit space-like vector orthogonal to the Lorentz plane spanned by $A$ and $B$, and $\operatorname{det} F=$ \pm 1 . To any null frame there is a uniquely determined associated orthonormal frame defined by

$$
L(F)=\left(\frac{1}{\sqrt{2}}(A+B), \frac{1}{\sqrt{2}}(A-B), C\right) .
$$

A null frame $F$ will be called proper if $L(F) \in \mathrm{SO}^{+}(1,2)$. One refers to

$$
N=\frac{1}{\sqrt{2}}\left[\begin{array}{ccc}
1 / \sqrt{2} & -1 / \sqrt{2} & 0 \\
-1 / \sqrt{2} & 1 / \sqrt{2} & 0 \\
0 & 0 & 1
\end{array}\right]
$$

as the "standard null frame": the associated orthonormal frame is the standard orthonormal frame $\left(e_{0}, e_{1}, e_{2}\right)$. More generally,

LEMMA (2.1). If $F$ is a null frame, then $L(F)=F \cdot N^{-1}$.

Proposition (2.2). The set of proper null frames is precisely the right coset of $\mathrm{SO}^{+}(1,2)$ in $\mathrm{GL}(3, \mathrm{R})$ determined by $N$.

Proposition (2.3). Let $s \mapsto F(s)$ be a curve in GL(3, R). Then $F(s) \in$ $\mathrm{O}(1,2) \cdot N$ if and only if $F\left(s_{0}\right) \in(1,2) \cdot N$ for some $s_{0} \in \mathbf{R}$ and $F^{-1} d F / d s$ has the form

$$
\left[\begin{array}{ccc}
k_{1} & 0 & k_{3} \\
0 & -k_{1} & k_{2} \\
k_{2} & k_{3} & 0
\end{array}\right]
$$

Remark. In Proposition (2.3), if, for some $s_{0} \in \mathbf{R}, F\left(s_{0}\right)$ is proper, then $F(s)$ is proper for all $s$.

A curve $s \mapsto F(s)$ in $\mathrm{SO}^{+}(1,2) \cdot N$ is a (proper) frame curve. If $F(s)=$ $(A(s), B(s), C(s))$ is a frame curve, then Proposition (2.3) implies that the following system of differential equations is satisfied: 


$$
\begin{aligned}
& d A / d s=k_{1}(s) A(s) \quad+k_{2}(s) C(s), \\
& d B / d s=-k_{1}(s) B(s)+k_{3}(s) C(s), \\
& d C / d s=k_{3}(s) A(s)+k_{2}(s) B(s)
\end{aligned}
$$

The equations which comprise (2.4) will be called the Frenet equations for $F$. It is clear that, given a proper null frame $F$ and a system (2.4), there is a unique frame curve $F(s)$ which satisfies $F(0)=F$ and has (2.4) as its Frenet equations.

A null curve in $\mathbf{L}^{3}$ is a curve $s \mapsto x(s)$ all of whose tangent vectors are nonzero and null $(\langle d x / d s, d x / d s\rangle=0)$. Since this condition is unaffected by a change of parameter, there is no analog of the arc-length parameter which curves in Euclidean space possess. There is, however, the following proposition about null curves.

Proposition (2.5). Let $x(s)$ be a null curve in $\mathbf{L}^{3}$ such that $x(0)=0$. Then $x(s)$ lies within the causal cone of $\mathbf{L}^{3}$.

Proof. If $x(s)=\left(x^{0}(s), x^{1}(s), x^{2}(s)\right)$ is the curve, then it is assumed that $x^{i}(0)=0(i=0,1,2)$ and

$$
\left(\frac{d x^{0}}{d s}\right)^{2}=\left(\frac{d x^{1}}{d s}\right)^{2}+\left(\frac{d x^{2}}{d s}\right)^{2}
$$

We may assume that $d x^{0} / d s>0$. Let $\tau(s)=\left(x^{1}(s), x^{2}(s)\right)$ be the projection of $x(s)$ onto the Euclidean plane generated by $\left\{e_{1}, e_{2}\right\}$. Then the arc length from $\tau(0)$ to $\tau(s)$ is

$$
\begin{aligned}
\int_{0}^{s}\left\|\tau^{\prime}(u)\right\| d u & =\int_{0}^{s}\left(\left(\frac{d x^{1}}{d u}\right)^{2}+\left(\frac{d x^{2}}{d u}\right)^{2}\right)^{1 / 2} d u \\
& =\int_{0}^{s} \frac{d x^{0}}{d u} d u=x^{0}(s) .
\end{aligned}
$$

This arc length must be at least the length of the line segment from $\tau(0)$ to $\tau(s)$, which is $\|\tau(s)\|=\left(\left(x^{1}(s)\right)^{2}+\left(x^{2}(s)\right)^{2}\right)^{1 / 2}$. Hence,

$$
0 \geqslant-\left(x^{0}(s)\right)^{2}+\left(x^{1}(s)\right)^{2}+\left(x^{2}(s)\right)^{2}
$$

which says that $x(s)$ lies in the causal cone of $\mathbf{L}^{3}$. Q.E.D.

A frame for a null curve $x(s)$ is a proper frame curve $F(s)=$ $(A(s), B(s), C(s))$ such that $d x / d s$ is a positive scalar multiple of $A(s) ; x(s)$ is said to be framed by $F(s)$. Frames for null curves are not unique: if $(A(s), B(s), C(s))$ frames a given null curve, so does $\left(\frac{1}{2}(A(s), 2 B(s), C(s))\right)$. Therefore, the curve and a frame must be given together. If $(x, F)$ is a framed 
null curve, then one has the Frenet equations (2.4) with $d x / d s=k_{0}(s) A(s)$, $k_{0}(s)>0$. If a point $y$, a proper null frame $F$, and functions $k_{i}(s)(i=$ $0,1,2,3)$ with $k_{0}(s)>0$ are specified, there is a unique framed null curve $(x(s), F(s))$ which satisfies $x(0)=y, F(0)=F$, and whose Frenet equations are (2.4) with $F=(A, B, C)$ and $d x / d s=k_{0}(s) A(s), k_{0}(s)>0$.

EXAMPLE (2.6). FRAMED NULL CURVE. Using coordinates with respect to the standard basis of $\mathbf{L}^{3}$, define $(x, F)$ as follows:

$$
F(s):\left\{\begin{array}{l}
x(s)=\left(\frac{4}{3} s^{3}+s, \frac{4}{3} s^{3}-s, 2 s^{2}\right), \\
A(s)=\left(4 s^{2}+1,4 s^{2}-1,4 s\right), \\
B(s)=\left(\frac{1}{2}, \frac{1}{2}, 0\right), \\
C(s)=(-2 s,-2 s,-1) .
\end{array}\right.
$$

The Frenet equations for $(x, F)$ are simple: let $k_{0}(s)=1, k_{2}(s)=-4$, and $k_{1}(s)=k_{3}(s)=0$.

In Example (2.6), $k_{0}(s)=1$ and $k_{1}(s) \equiv 0$. Thus $A(s)$ is the tangent vector (rather than its direction) and $C(s)$ is analogous to the principal normal in the standard Frenet frame for a curve in $\mathbf{E}^{3}$. More generally, a Cartan-framed curve is a framed null curve $(x(s), F(s))$ whose Frenet equations have the form

$$
\begin{gathered}
\frac{d x}{d s}=A(s), \quad \frac{d A}{d s}=k_{2}(s) C(s), \quad \frac{d B}{d s}=k_{3}(s) C(s), \\
\frac{d C}{d s}=k_{3}(s) A(s)+k_{2}(s) B(s) .
\end{gathered}
$$

Bonnor [B] has made a thorough study of null curves in $\mathbf{L}^{4}$, and his methods may be adapted to examine null curves in $\mathbf{L}^{3}$ (or in $\mathbf{L}^{n}$, for any $n \geqslant 2)$.

3. Theory of Lorentz hypersurfaces in $\mathbf{L}^{n+1}$. A hypersurface $M$ in $\mathbf{L}^{n+1}$ is a Lorentz hypersurface if the tangent space to $M$ at each point of $M$ inherits a Lorentz metric from $\mathbf{L}^{n+1}$. If $f: M \rightarrow \mathbf{L}^{n+1}$ is an isometric immersion, then $f(M)$ is a Lorentz hypersurface which may simply be denoted by $M$. The Lorentz metric on $M, f(M)$, or $\mathbf{L}^{n+1}$ will be denoted by $\langle$,$\rangle ; the context will$ resolve any ambiguity. Each point of a Lorentz hypersurface $M$ has a neighborhood (in $M$ ) on which is defined a vector field, usually denoted by $\xi$, consisting of space-like unit normals.

Let $D$ be the usual (flat) connection on $\mathbf{L}^{n+1}$. The Levi-Civita connection $\nabla$ on $M$ is specified by the Gauss formula

$$
D_{X} Y=\nabla_{X} Y+h(X, Y) \xi
$$

where $X$ and $Y$ are tangent vector fields on $M$, and $\xi$ is a local (space-like) 
unit normal field on $M$; if $x \in M$, then $\left(\nabla_{X} Y\right)_{x}$ is the tangential component of $\left(D_{X} Y\right)_{x}$ (cf. [KN, Vol. II, Chapter VII]).

In (3.1), $h$ is a symmetric bilinear form, the second fundamental form. There is a field $A$ of endomorphisms of $T(M)$, symmetric with respect to the Lorentz metric, such that

$$
D_{X} \xi=-A X
$$

and

$$
\langle A X, Y\rangle=h(X, Y) \text {. }
$$

The curvature tensor $R$ is related to the second fundamental tensor by the equation of Gauss:

$$
R(X, Y)=A X \wedge A Y
$$

where $X \wedge Y$ denotes the endomorphism of the tangent space defined by

$$
(X \wedge Y) Z=\langle Z, Y\rangle X-\langle Z, X\rangle Y \text {. }
$$

The second fundamental tensor satisfies the equation of Codazzi:

$$
\left(\nabla_{X} A\right) Y=\left(\nabla_{Y} A\right) X .
$$

Finally, for each $x \in M, T_{0}(x)=\operatorname{ker} A_{x}=\left\{x \in T_{x} M:(A X)_{x}=0\right\}$ is called the relative nullity space at $x$.

An important fact about $R$ is the following.

Proposition (3.6). $R \equiv 0$ (that is, $R(X, Y)=0$ for all $X, Y$ ) if and only if $M$ is locally isomorphic with $\mathbf{L}^{n}$.

Proof. This is analogous to the proof in the Riemannian case (cf. [S, Vol. II, pp. 6-21]), with $\left\langle X^{1}, X^{1}\right\rangle=-1$. Q.E.D.

Let us consider some examples of Lorentz hypersurfaces.

(3.7). $M \times \mathbf{R}$. Let $M$ be a Riemannian manifold with metric $g$. If $\mathbf{R}$ is the real line, then define a Lorentz metric on the direct product $M \times \mathbf{R}$ as follows. A tangent vector to $M \times \mathbf{R}$ has the form $(X, a \partial / \partial t)$, where $X$ is tangent to $M$ and $a \in \mathbf{R}$. The metric is defined by

$$
\langle(X, a),(Y, b)\rangle=g(X, Y)-a b .
$$

Notice that if $M$ lies in $\mathbf{E}^{n}$, then $M \times \mathbf{R}$ lies in $\mathbf{L}^{n+1}$.

(3.8). Cylinder over a time-like curve. Let $c(t)$ be a unit speed time-like curve in $\mathbf{L}^{2}$. Let $\mathbf{E}^{1}$ denote $\mathbf{R}$ with its usual (positive definite) metric $(d t)^{2}$, and let $\mathbf{L}^{1}$ denote $\mathbf{R}$ with the negative definite metric $-(d t)^{2}$. Then $c$ is an isometric immersion: $\mathbf{L}^{1} \rightarrow \mathbf{L}^{2}$, and, if the products are Riemannian direct products,

$$
c \times \text { id: } \mathbf{L}^{1} \times \mathbf{E}^{1} \rightarrow \mathbf{L}^{2} \times \mathbf{E}^{1}
$$

defines an isometric immersion: $\mathbf{L}^{2} \rightarrow \mathbf{L}^{3}$. 
(3.9). $B$-scroll. Let $(x(s), F(s))$ be a Cartan-framed null curve in $\mathbf{L}^{3}$. The $B$-scroll of $(x, F)$ is parametrized by $f(s, u)=x(s)+u B(s)$. Since $f_{*}(\partial / \partial u)$ $=B(s)$ and $f_{*}(\partial / \partial s)=A(s)+u k_{3}(s) C(s)$, the metric at $f(s, u)$ has the matrix $\left[-_{-1}^{a}{ }_{a}^{-1}\right]$, of determinant -1 , with respect to the basis $\left\{f_{*}(\partial / \partial s)\right.$, $\left.f_{*}(\partial / \partial u)\right\}$. Thus, the metric is Lorentz.

There is a relationship between $\boldsymbol{B}$-scrolls and flat Lorentz surfaces, which is described by the following theorem.

THEOREM (3.10). The $B$-scroll is flat if and only if $k_{3}(s)=0$ for all $s$.

Proof. If $k_{3} \equiv 0$, let $\partial / \partial s=(1 / \sqrt{2})\left[\begin{array}{c}1 \\ -1\end{array}\right]$ and $\partial / \partial u=(1 / \sqrt{2})\left[\begin{array}{l}1 \\ 1\end{array}\right]$ form a null frame for $\mathbf{L}^{2}$. Then $f$ describes a local isometry between the $B$-scroll and $\mathbf{L}^{2}$ (see (3.9)).

Conversely, suppose the $B$-scroll is flat; then its curvature tensor is identically zero. Now, there are the equalities:

$$
\begin{gathered}
\left\langle\nabla_{u} \frac{\partial}{\partial u}, \frac{\partial}{\partial u}\right\rangle=\left\langle\nabla_{s} \frac{\partial}{\partial u}, \frac{\partial}{\partial u}\right\rangle=\left\langle\nabla_{u} \frac{\partial}{\partial u}, \frac{\partial}{\partial s}\right\rangle=0, \\
\left\langle\nabla_{s} \frac{\partial}{\partial u}, \frac{\partial}{\partial s}\right\rangle=u k_{3}(s)^{2} .
\end{gathered}
$$

That $\langle\partial / \partial u, \partial / \partial s\rangle=-1$ implies that $\nabla_{s} \partial / \partial u=-u\left(k_{3}(s)\right)^{2} \partial / \partial u$; and hence,

$$
\begin{aligned}
R\left(\frac{\partial}{\partial u}, \frac{\partial}{\partial s}\right) \frac{\partial}{\partial u} & =\nabla_{u} \nabla_{s} \frac{\partial}{\partial u}-\nabla_{s} \nabla_{u} \frac{\partial}{\partial u} \\
& =-\nabla_{u}\left(u k_{3}(s)^{2} \frac{\partial}{\partial u}\right)=-k_{3}(s)^{2} \frac{\partial}{\partial u}
\end{aligned}
$$

If $R \equiv 0$ at all points of the $B$-scroll, then $k_{3}(s)=0$ for all $s$. Q.E.D.

Thus, $B$-scrolls are Lorentz surfaces which are flat just in the case that the $B$-direction is parallel. Notice also in this case that $C(s)$ is a unit (space-like) normal which is parallel along the $B$-direction; it follows that $f_{*}(\partial / \partial u)$ generates the relative nullity space at each point $f(s, u)$ such that $k_{2}(s) \neq 0$ (cf. (3.11) below).

A Cartan-framed null curve whose $B$-direction is parallel will be called a generalized null cubic $(G N C)$. The name can be justified as follows. By definition, the Frenet equations of a GNC $(x(s), F(s))$ have the form

$$
\frac{d x}{d s}=A(s), \quad \frac{d A}{d s}=k_{2}(s) C(s), \quad \frac{d B}{d s}=0, \quad \frac{d C}{d s}=k_{2}(s) B(s)
$$

If $k_{2}(s)=1$ for all $s$, then the curve $x(s)$ with initial condition $x(0)=0$ and $F(0)=N$ is easily seen to be 


$$
x(s)=\left[\begin{array}{c}
\frac{1}{2}\left(s+\frac{1}{6} s^{3}\right) \\
-\frac{1}{2}\left(s-\frac{1}{6} s^{3}\right) \\
\frac{1}{2} s^{2}
\end{array}\right]
$$

Bonnor [B] calls such a curve a null cubic. It is from this that the present term "generalized null cubic" derives.

Example (2.6) is in fact a GNC. Therefore, Theorem (3.10) implies that

$$
f(s, u)=\left(\frac{u}{2}+\frac{4}{3} s^{3}+s, \frac{u}{2}+\frac{4}{3} s^{3}-s, 2 s^{2}\right)
$$

describes a flat Lorentz surface in $\mathbf{L}^{3}$. (Here, of course, $(s, u)$ are coordinates with respect to a null frame for $L^{2}$, so

$$
\left\langle\frac{\partial}{\partial s}, \frac{\partial}{\partial s}\right\rangle=\left\langle\frac{\partial}{\partial u}, \frac{\partial}{\partial u}\right\rangle=0 \text { and }\left\langle\frac{\partial}{\partial u}, \frac{\partial}{\partial s}\right\rangle=-1 ;
$$

the $\mathbf{L}^{3}$ coordinates are with respect to the standard orthonormal basis.)

\section{PART II. COMPLETENESS OF THE RELATIVE NULLITY FOLIATION}

4. Preliminary results. We consider isometric immersions: $\mathbf{L}^{n} \rightarrow \mathrm{L}^{n+1}$. It follows from the equation of Gauss (3.4) that the dimension $\nu_{0}(x)$ of the relative nullity space at each $x \in \mathbf{L}^{n}$ is $n-1$ or $n$. If $\nu_{0}(x)=n$, then $x$ is an umbilic (or planar) point. Define:

$$
W=\left\{x \in \mathbf{L}^{n}: x \text { is not an umbilic point }\right\} .
$$

Proposition (4.1). $W$ is an open subset of $\mathbf{L}^{n}$.

Proof. $W=\left\{x \in \mathbf{L}^{n}: A_{x} \equiv 0\right\}$. Q.E.D.

Let $x \in W$. Then $T_{0}(x)$ is an $(n-1)$-dimensional subspace of $\mathbf{L}_{x}^{n}=$ $T_{x}\left(\mathbf{L}^{n}\right)$. Therefore, by Theorem (1.1), exactly one of the following statements is true:

(4.2) (i) $T_{0}(x)$ is an $(n-1)$-dimensional Lorentzian subspace,

(ii) $T_{0}(x)$ is an $(n-1)$-dimensional Euclidean subspace,

(iii) $T_{0}(x) \simeq E \oplus \operatorname{Span}\{\xi\}$ where $E$ is an $(n-2)$-dimensional Euclidean subspace, $\xi$ is a light-like vector, and $\langle Y, \xi\rangle=0$ for all $Y \in E$.

In cases (i) and (ii) of (4.2) the metric inherited by $T_{0}(x)$ is nondegenerate (with index 1 and 0 , respectively); in case (iii), the inherited metric is degenerate. We will say that the relative nullity (space) at $x$ is degenerate or nondegenerate according to whether the inherited metric is degenerate or nondegenerate.

TheOREM (4.3). Let $x \in W$. The following statements are equivalent:

(i) $T_{0}(x)$ is degenerate,

(ii) the image of $A_{x}$ is a light line,

(iii) $A_{x}^{2} \equiv 0$. 
Proof. (i) $\Rightarrow$ (ii). $\quad T_{0}(x) \simeq E \oplus \operatorname{Span}\{\xi\}$ per (4.2)(iii), so $\mathbf{L}_{x}^{n}=T_{0}(x) \oplus$ $\operatorname{Span}\{\eta\}$, a direct but not orthogonal sum in which $\operatorname{Span}\{\xi, \eta\}$ is the Lorentz plane orthogonal to $E$ in $\mathbf{L}_{x}^{n}$. Since $x \in W, A \eta \neq 0$. Clearly, $A \eta$ is orthogonal to $E$. But also $\langle A \eta, \xi\rangle=\langle\eta, A \xi\rangle=0$. Since the only vectors in a Lorentz plane orthogonal to a nonzero light vector are linearly dependent vectors (see [G]), $\operatorname{Span}\{A \eta\}=\operatorname{Span}\{\xi\}$.

(ii) $\Rightarrow$ (iii). If $\eta \in \mathbf{L}_{x}^{n}$ and $\mathbf{L}_{x}^{n}=T_{0}(x) \oplus \operatorname{Span}\{\eta\}$, then $\left\langle A^{2} \eta, Y\right\rangle=$ $\langle A \eta, A Y\rangle=0$ for all $Y \in T_{0}(x)$, and $\left\langle A^{2} \eta, \eta\right\rangle=\langle A \eta, A \eta\rangle=0$. Thus, $A^{2} \eta$ $=0$.

(iii) $\Rightarrow$ (i). If $A_{x}^{2} \equiv 0$, then the image of $A_{x}$ lies in $T_{0}(x)$. Suppose the image of $A_{x}$ is $\operatorname{Span}\{A \eta\}\left(\eta \in \mathbf{L}_{x}^{n}\right)$. If $Y \in T_{0}(x)$ then $\langle A \eta, Y\rangle=\langle\eta, A Y\rangle=$ 0 . Thus, $A \eta$ is a vector in $T_{0}(x)$ which is orthogonal to every vector in $T_{0}(x)$. Q.E.D.

Corollary (4.4). $G=\left\{x \in W: T_{0}(x)\right.$ is nondegenerate $\}$ is an open subset of $W$ (and hence of $\mathbf{L}^{n}$ ).

Proof. By Theorem (4.3), $G=\left\{x \in W: A_{x}^{2} \neq 0\right\}$. Q.E.D.

A foliation of an open subset of a manifold is an integrable differentiable distribution on the subset. A differentiable distribution-in particular, a foliation-is totally geodesic if $\nabla_{X} Y$ lies in the distribution whenever $X$ and $Y$ do. We remark that parallel transport along curves whose tangent vectors lie in a totally geodesic foliation preserves the foliation. The maximal connected integral submanifolds corresponding to a foliation according to the theorem of Frobenius are called the leaves of the foliation. A totally geodesic foliation is called complete if any affinely-parametrized geodesic which is tangent to the foliation can be extended to all values of the parameter and still lie in a leaf of the foliation.

On $W$, there is an $(n-1)$-dimensional distribution $T_{0}: x \rightarrow T_{0}(x)$, called the relative nullity distribution. The present purpose is to show that $T_{0}$ is a complete totally geodesic foliation of $W$. First, we will show that $T_{0} \mid G$ is a complete totally geodesic foliation of $G$, using methods appearing in $\left[\mathbf{N}_{\mathbf{1}}\right]$ and $\left[\mathbf{N}_{2}\right]$, which derive from those in $[\mathbf{A}]$ and [F]. After showing that $W \backslash G$ is open in $W$ (indeed, we shall ultimately see that $G=\varnothing$ or $G=W$ ), we will then show that $T_{0} \mid(W \backslash G)$ is a complete totally geodesic foliation of $W \backslash G$. It will then be apparent that $T_{0}$ is a complete totally geodesic foliation of $W$.

5. Nondegenerate relative nullities. In this section, we consider the restriction of the relative nullity distribution to $G$. Therefore, for each $x \in G$, $T_{0}(x)$ is a Lorentz or Euclidean $(n-1)$-dimensional subspace of $\mathbf{L}_{x}^{n}$, and there is a uniquely determined orthogonal complement to $T_{0}(x)$ in $\mathbf{L}_{x}^{n}$, which we will denote by $T_{0}^{\perp}(x)$. 
Proposition (5.1). $T_{0}^{\perp}(x)$ is a nontrivial eigenspace for $A_{x}$.

Proof. $T_{0}^{\perp}(x)$ is generated by a nonzero vector $Z$ such that $\langle Z, Z\rangle \neq 0$. If $Y \in T_{0}(x)$, then $0=\left\langle Z, A_{x} Y\right\rangle=\left\langle A_{x} Z, Y\right\rangle$, so that there is some $\lambda(x) \neq 0$ such that $A_{x} Z=\lambda(x) Z$. Clearly, $\lambda(x)$ is independent of the choice of $Z$. Q.E.D.

REMARK. Since $\lambda(x)$ is the nonzero eigenvalue of $A_{x}$ for each $x \in G$, the function $\lambda$ is differentiable.

Proposition (5.2). $T_{0}$ and $T_{0}^{\perp}$ are differentiable distributions on $G$.

Proof. Lemma 1 of $\left[\mathbf{N}_{1}\right]$. Q.E.D.

Proposition (5.3). $T_{0}$ is integrable.

Proof. Lemma 2 of $\left[\mathbf{N}_{\mathbf{1}}\right]$. Q.E.D.

Now suppose $X$ is a vector field belonging to $T_{0}(x)$ and $Z$ is a vector field belonging to $T_{0}^{\perp}$. The Codazzi equation (3.5) implies

$$
\nabla_{X}(A Z)-A\left(\nabla_{X} Z\right)=\nabla_{Z}(A X)-A\left(\nabla_{Z} X\right) .
$$

Suppose a tangent vector $U$ is decomposed into $T_{0^{-}}$and $T_{0}^{\perp}$-components: $U=U_{0}+U^{\perp}$. Then $A U=\lambda U^{\perp}$ and $(A-\lambda I) U=-\lambda U_{0}$. Since $A X=0$ and $A Z=\lambda Z,(5.4)$ implies

$$
(X \lambda) Z+\lambda\left(\nabla_{X} Z\right)_{0}=-\lambda\left(\nabla_{Z} X\right)^{\perp}
$$

Setting the $T_{0}$ - and $T_{0}{ }^{\perp}$-components on each side of (5.5) equal yields these useful equations:

$$
\begin{gathered}
(X \cdot \lambda) Z+\lambda\left(\nabla_{Z} X\right)^{\perp}=0 \\
\lambda\left(\nabla_{X} Z\right)_{0}=0 .
\end{gathered}
$$

Since $\lambda \neq 0$, (5.7) implies that $\nabla_{X} Z \in T_{0}{ }^{\perp}$. Now, if $Y$ is a vector field belonging to $T_{0}$, then $\langle Y, Z\rangle=0$, and so

$$
0=X \cdot\langle Y, Z\rangle=\left\langle\nabla_{X} Y, Z\right\rangle+\left\langle Y, \nabla_{X} Z\right\rangle=\left\langle\nabla_{X} Y, Z\right\rangle \text {. }
$$

Thus, we have

Proposition (5.8). $T_{0}$ is a totally geodesic foliation of $G$.

If $x \in G$, denote the leaf of the relative nullity foliation through $x$ by $M_{0}(x)$. Proposition (5.8) implies that $M_{0}(x)$ is a piece of a hyperplane in $\mathbf{L}^{n}$ through $x$, since totally geodesic submanifolds of any $\mathbf{R}^{m}$ with the flat connection are pieces of hyperplanes.

Now let $x_{0} \in G$ and suppose $x_{t}$ is an affinely-parametrized geodesic which lies in $M_{0}\left(x_{0}\right)$ for $0 \leqslant t<b$. Since $\mathbf{L}^{n}$ is complete, $x_{t}$ can be extended indefinitely in $\mathbf{L}^{n}$. To show that $T_{0}$ is a complete foliation of $G$, it suffices to show that for all $t, x_{t} \in M_{0}\left(x_{0}\right)$. 
Lemma (5.9). If $x_{b} \in W$, then there exists a positive $\varepsilon$ such that $x_{t} \in M_{0}\left(x_{0}\right)$ $\leqslant G$ for $0 \leqslant t<b+\varepsilon$.

Proof. First, since $T_{0}$ and $T_{0}^{\perp}$ are parallel along the $T_{0}$-curve $x_{t}$, we may choose orthonormal parallel vector fields $Y_{t}^{i}(i=1, \ldots, n-1)$ along $x_{t}$ such that $T_{0}\left(x_{t}\right)=\operatorname{Span}\left\{Y_{t}^{1}, \ldots, Y_{t}^{n-1}\right\}$. Extend these parallelly (in $\mathbf{L}^{n}$ ) beyond $t=b$. Then for all $t, \operatorname{Span}\left\{Y_{t}^{1}, \ldots, Y_{t}^{n-1}\right\}$ is an $(n-1)$-dimensional subspace of $\mathbf{L}_{x_{i}}^{n}$. Moreover, $A Y_{b}^{j}=\lim _{t \rightarrow b} A Y_{t}^{j}=0$ for $j=1, \ldots, n-1$, so $\operatorname{Span}\left\{Y_{b}^{1}, \ldots, Y_{b}^{n-1}\right\} \subseteq T_{0}\left(x_{b}\right)$. If $x_{b} \in W$, these spaces have the same dimension and so coincide. The metric is preserved by parallel transport, so $T_{0}\left(x_{b}\right)$ has the same metric as $T_{0}\left(x_{t}\right)$ for $t<b$. In this case, that metric is nondegenerate, so $x_{b} \in G$. By Corollary (4.4), $x_{t}$ must lie in $G$ for parameters $t$ in some neighborhood of $b$. Now choose coordinates $y^{i}$ about $x_{b}$ such that the integral submanifolds of $T_{0}$ are given by $y^{n}=$ constant. In particular, $M_{0}\left(x_{b}\right)$ is the slice $y^{n}=0$. The geodesic $x_{t}$, for $t<b$ at least, lies in a slice $y^{n}=c$ which is $M_{0}\left(x_{0}\right)$ near $x_{b}$. But $c=\lim _{t \rightarrow b} x_{t}^{n}=x_{b}^{n}=0$. Thus $M_{0}\left(x_{0}\right)$ and $M_{0}\left(x_{b}\right)$ must coincide, and $M_{0}\left(x_{0}\right)$ is an open hyperplane piece containing $x_{b}$. Q.E.D.

As a consequence of Lemma (5.9), it is imperative to show that $x_{b} \in W$ if $x_{t}$ is the geodesic described above. To this end, let $y$ be a point on the geodesic: $y=x_{t_{1}}, 0 \leqslant t_{1}<b$. Let $X$ be an extension of the tangent vector field $\vec{x}_{t}$ to a $T_{0}$-field near $y$. If $Z_{t}$ is a $T_{0}^{\perp}$-field along $x_{t}$, let $Z$ be an extension of $Z_{t}$ to a $T_{0}^{\perp}$-field near $y$. Define $P: T_{0} \times T_{0}^{\perp} \rightarrow T_{0}^{\perp}$ near $y$ by $P(X, Z)=$ $\left(\nabla_{Z} X\right)^{\perp}$. (For the purposes of the definition, $X$ and $Z$ may be arbitrary $T_{0^{-}}$ and $T_{0}$-fields, respectively, near $y$.)

Lemma (5.10). If $u$ is a point near $y$, then $P$ gives a bilinear map

$$
P_{u}: T_{0}(u) \times T_{0}^{\perp}(u) \rightarrow T_{0}^{\perp}(u) .
$$

Proof. Choose coordinates $x^{i}$ near $u$ so that

$$
T_{0}=\operatorname{Span}\left\{\partial / \partial x^{1}, \ldots, \partial / \partial x^{n-1}\right\}
$$

If $X=\sum_{j=1}^{n-1} X^{j} \partial / \partial x^{j}$, then

$$
\begin{aligned}
\left(\nabla_{Z} X\right)^{\perp} & =\left[\sum_{j=1}^{n-1}\left(Z \cdot X^{j}\right)\left(\frac{\partial}{\partial x^{j}}\right)+\sum_{j=1}^{n-1} X^{j}\left(\nabla_{Z} \frac{\partial}{\partial x^{j}}\right)\right]^{\perp} \\
& =\sum_{j=1}^{n-1} X^{j}\left(\nabla_{Z} \frac{\partial}{\partial x^{j}}\right)^{\perp} .
\end{aligned}
$$

Thus, if $X_{u}=0$ or $Z_{u}=0$, then $\left(\nabla_{Z} X\right)_{u}^{\perp}=0$. Q.E.D.

LEMMA (5.11). If there is a vector field $Z_{t}$ along $x_{t}$ for $0 \leqslant t \leqslant b$ such that $Z_{t} \in T_{0}^{\perp}\left(x_{t}\right)$ and $\nabla_{t} Z_{t}=P\left(X, Z_{t}\right)_{x_{t}}$ for $0 \leqslant t<b$, then $x_{b} \in W$. 
Proof. Let $Y_{t}$ be a parallel field along $x_{t}$ such that $Y_{b} \in T_{0}\left(x_{b}\right)$. For $t<b$,

$$
\begin{aligned}
\frac{d}{d t}\left\langle A Z_{t}, Y_{t}\right\rangle & =\left\langle\nabla_{t}\left(\lambda Z_{t}\right), Y_{t}\right\rangle=\left\langle(X \lambda) Z+\lambda\left(\nabla_{t} Z_{t}\right), Y_{t}\right\rangle \\
& =\left\langle(X \lambda) Z+\lambda\left(\nabla_{Z} X\right)^{\perp}, Y_{t}\right\rangle=0
\end{aligned}
$$

by (5.6). Since $\left\langle A Z_{b}, Y\right\rangle=\left\langle Z_{b}, A Y_{b}\right\rangle=0$, it follows that $\left\langle A Z_{t}, Y_{t}\right\rangle=0$ for all $t \in[0, b]$, in particular for $t=0$, but $\langle A Z, Y\rangle_{x_{0}}=\lambda\left(x_{0}\right)\langle Z, Y\rangle_{x_{0}}$, which, since $\lambda\left(x_{0}\right) \neq 0$, implies that $\left\langle Z_{0}, Y_{0}\right\rangle=0$. Thus, $Y_{0}$ is orthogonal to $T_{0}^{\perp}\left(x_{0}\right)$; i.e., $Y_{0} \in T_{0}\left(x_{0}\right)$. Therefore, the parallel transport isomorphism maps the $(n-1)$-dimensional $T_{0}\left(x_{0}\right)$ onto $T_{0}\left(x_{b}\right)$, so $\operatorname{dim} T_{0}\left(x_{b}\right)=n-1 ; x_{b} \in W$. Q.E.D.

LEMMA (5.12). A vector field $Z_{t}$ exists along $x_{t}$ which satisfies the hypotheses of Lemma (5.11).

Proof. Suppose $Z_{t}$ is a solution to the differential equation $\nabla_{t} Z_{t}=$ $P_{x_{t}}\left(\vec{x}_{t}, Z_{t}\right)$ along $x_{t}$ satisfying a $T_{0}^{\perp}$ initial condition at $t=0$. If $y=x_{t_{1}}$ is a point on the geodesic under consideration, then let $X$ and $Z$ be the extensions of $\vec{x}_{t}$ and $Z_{t}$ near $y$ mentioned above. Then at each $y$ on the geodesic, the equation $\nabla_{X} Z=P(X, Z)$ holds. Therefore,

$$
\nabla_{X}^{2} Z=\nabla_{X}(P(X, Z))=\nabla_{X}\left(\nabla_{Z} X\right)^{\perp}=\left(\nabla_{X} \nabla_{Z} X\right)^{\perp}
$$

the last equality holds because $X \in T_{0}$ and $T_{0}$ is totally geodesic. Now, since $R \equiv 0$ and there is no torsion $\left([X, Z]=\nabla_{X} Z-\nabla_{Z} X\right)$, we get

$$
\nabla_{X}^{2} Z=\left(\nabla_{Z} \nabla_{X} X\right)^{\perp}+\left(\nabla_{\nabla_{X}} X\right)^{\perp}-\left(\nabla_{\nabla_{Z} X} X\right)^{\perp} \text {. }
$$

Since $X \in T_{0}, \nabla_{X} X \in T_{0}$, and in particular $\left(\nabla_{X} X\right)_{y}=\left(\nabla_{t} \vec{x}_{t}\right)_{t_{1}}=0$. So if $Y \in T_{0}^{\perp}$,

$$
\left\langle\nabla_{Z} \nabla_{X} X, Y\right\rangle_{y}=Z \cdot\left\langle\nabla_{X} X, Y\right\rangle_{y}-\left\langle\left(\nabla_{X} X\right)_{y}, \nabla_{Z} Y\right\rangle=0 .
$$

Thus, along the geodesic, $\left(\nabla_{Z} \nabla_{X} X\right)^{\perp}=0$. Since $X \in T_{0}$ and $Z \in T_{0}^{\perp}$, $\nabla_{X} Z \in T_{0}^{\perp}$ (see (5.7)) and so by definition $\left(\nabla_{\nabla_{X} Z} X\right)^{\perp}=P\left(X, \nabla_{X} Z\right)$. Finally,

$$
\begin{aligned}
\left(\nabla_{\nabla_{z} X} X\right)^{\perp} & =\left(\nabla_{\left.\left(\nabla_{z} X\right)_{0}+\left(\nabla_{z} X\right)^{\perp} X\right)^{\perp}}\right. \\
& =\left(\nabla_{\left(\nabla_{z} X\right)_{0} X}\right)^{\perp}+\left(\nabla_{\left.\left(\nabla_{z} X\right)^{\perp} X\right)^{\perp}}\right. \\
& =\left(\nabla_{\left(\nabla_{z} X\right)} X\right)^{\perp}=P(X, P(X, Z)) .
\end{aligned}
$$

With this information, (5.13) becomes (along the geodesic)

$$
\nabla_{X}^{2} Z=P\left(X, \nabla_{X} Z\right)-P(X, P(X, Z))=P\left(X, \nabla_{X} Z-P(X, Z)\right)=0 .
$$

Now, since $P: T_{0} \times T_{0}^{\perp} \rightarrow T_{0}^{\perp}$ is bilinear, the linear differential equation $\nabla_{t} Z_{t}=P\left(\vec{x}_{t}, Z_{t}\right)$ admits a unique solution $Z_{t}, 0<t<b$, corresponding to any initial condition $Z_{0} \in T_{0}^{\perp}\left(x_{0}\right)$. We have just seen that such a solution 
must satisfy $\nabla_{t}^{2} Z_{t}=0$. If $\mathscr{Z}$ is a parallel $T_{0}^{\perp}$-field along $x_{t}, 0 \leqslant t<b$, then, for some smooth $\Phi: \mathbf{R} \rightarrow \mathbf{R}$, we have $Z_{t}=\Phi(t) \mathscr{Z}$ and $d^{2} \Phi / d t^{2}=0$. Therefore, $\Phi(t)=\alpha t+\beta$, where the constants $\alpha$ and $\beta$ are determined by

$$
\begin{gathered}
Z_{0}=\beta \mathscr{Z}, \\
\alpha \mathscr{Z}=\left(\nabla_{t} Z_{t}\right)_{t=0}=\left.P\left(\vec{x}_{t}, Z_{t}\right)\right|_{t=0}=P\left(\vec{x}_{0}, \beta \mathscr{Z}\right) .
\end{gathered}
$$

Since $\mathscr{Z}$ can be extended as a parallel vector field along $x_{t}$ to $t=b$ and beyond, the vector field $Z_{t}=(\alpha t+\beta) \mathcal{Z}$ can be extended to beyond $t=b$ as well. If $0 \leqslant t<b$, then $Z_{t} \in T_{0}^{\perp}\left(x_{t}\right)$. Q.E.D.

THEOREM (5.14). $T_{0}$ is a complete totally geodesic foliation of $G$.

Proof. There is to show that if $x_{0} \in G$ and $x_{t}$ is a geodesic emanating from $x_{0}$ in a $T_{0}$ direction and extended indefinitely in the complete $\mathbf{L}^{n}$, then $x_{t} \in M_{0}\left(x_{0}\right) \subseteq G$ for all $t$. Let $b$ be defined by

$$
b=\sup \left\{u: x_{t} \in M_{0}\left(x_{0}\right) \text { for } 0 \leqslant t<u\right\} .
$$

Then $b>0$. Suppose $b$ is finite. Then, by Lemma (5.12), choose a vector field along $x_{t}$ such that, for $0 \leqslant t<b, Z_{t} \in T_{0}^{\perp}\left(x_{t}\right)$ and $\nabla_{t} Z_{t}=P\left(\vec{x}_{t}, Z_{t}\right)$. By Lemma (5.11), $x_{b} \in W$. But now Lemma (5.9) implies that for some positive $\varepsilon, x_{t} \in M_{0}\left(x_{0}\right)$ for $0 \leqslant t<b+\varepsilon$. This contradicts the nature of $b$ as a supremum. Therefore, $b$ is infinite. Q.E.D.

Corollary (5.15). For each $x \in G, M_{0}(x)$ is a hyperplane contained in $G$.

COROLlARY (5.16). The open set $G$ is a union of parallel hyperplanes (with metric of the same signature).

If $x \in G$ and/or $x \in W$, let $G_{x}$ (respectively, $W_{x}$ ) denote the component of $x$ in $G$ (respectively, in $W$ ).

Proposition (5.17). If $x \in G$, then $G_{x}=W_{x}$.

Proof. Suppose $x_{t}$ is a curve from $x_{0}=x$ to $x_{b}=z$ in $W_{x}$ such that $x_{t} \in G_{x}$ for each $t \in[0, b)$. Then (5.16) implies that $\left\{T_{0}(x): t \in[0, b)\right\}$ is a family of parallel nondegenerate tangent hyperplanes. Therefore, there are vector fields $Y_{t}^{i}(i=1, \ldots, n-1)$ along $x_{t}, 0 \leqslant t<b$, such that, for $t \in$ $[0, b)$

(i) $T_{0}\left(x_{t}\right)=\operatorname{Span}\left\{Y_{t}^{1}, \ldots, Y_{t}^{n-1}\right\}$,

(ii) $\left\langle Y_{t}^{i}, Y_{t}^{j}\right\rangle=\delta_{i}^{j}$ (with $\delta_{1}^{1}=-1$ if $T_{0}$ 's are Lorentz),

(iii) $\nabla_{t} Y_{t}^{i}=0, i=1, \ldots, n-1$.

Extend each $Y_{t}^{i}$ parallelly along $x_{t}$. Since $\left\langle Y_{t}^{i}, Y_{t}^{j}\right\rangle$ is preserved, $\operatorname{Span}\left\{Y_{b}^{1}, \ldots, Y_{b}^{n-1}\right\}$ is a nondegenerate tangent hyperplane through $z$. Also $A Y_{b}^{i}=\lim _{t \rightarrow b} A Y_{t}^{i}=0$, so this hyperplane lies in $T_{0}(z)$. Since $z \in W_{x}, T_{0}(z)$ has dimension $n-1$, and therefore coincides with the nondegenerate 
hyperplane $\operatorname{Span}\left\{Y_{b}^{1}, \ldots, Y_{b}^{n-1}\right\}$. As a result, $z \in G_{x}$. It follows from this argument that if $x_{t}$ is any curve in $W_{x}$ emanating from $x \in G_{x}$, then $\sup \{u$ : $x_{t} \in G_{x}$ for $\left.t \in[0, u)\right\}$ is not finite. Q.E.D.

6. Degenerate relative nullities. Now we consider $W \backslash G$, the subset of points $y \in W$ such that $T_{0}(y)$ carries a nondegenerate metric. By (4.2)(iii), we have at each such $y$ a decomposition

$$
T_{0}(y)=E(Y) \oplus \operatorname{Span}\{\xi\}
$$

where $\xi$ is a light vector and $E(y)$ is an $(n-2)$-dimensional Euclidean subspace of $\mathbf{L}_{y}^{n}$ orthogonal to $\xi$. Notice that although the light line in $T_{0}(y)$ is unique, the Euclidean "complement" $E(y)$ is not. Since $\mathbf{L}^{n}$ is locally connected, (5.17) implies

Proposition (6.1). $W \backslash G$ is open.

Now define a distribution on the open set $W \backslash G$ by $y \mapsto T_{0}(y)$. It is, of course, the restriction of the relative nullity foliation to the open set $W \backslash G$.

Proposition (6.2). $T_{0}$ is a differentiable distribution on $W \backslash G$.

Proof. Consider a point $y$ of $W \backslash G$, and select a local light field $L$ at $y$-i.e., a vector field $L$ in a neighborhood of $y$ such that $\langle L, L\rangle=0$-which satisfies $(A L)_{y} \neq 0$. Then $A L \neq 0$ near $y$ and, by (4.3)(ii), $A L$ is a local light field at $y$.

Indeed, for those $x$ near $y, A L_{x}$ lies in the light line of $T_{0}(x)$, and so $A L_{x} \perp T_{0}(x)$. If also $A L_{x} \perp L_{x}$, then $A L_{x} \perp \mathbf{L}_{x}^{n}$, implying $A L_{x}=0$, a contradiction if $x$ is close to $y$. So $\left\langle A L_{x}, L_{x}\right\rangle \neq 0$. Now one can compute directly that $\{A L+L, A L-L\}$ is an orthogonal set of two vectors, one space-like and one time-like, which therefore spans a Lorentz plane $\mathbf{L}^{2}(x)$ in $\mathbf{L}_{x}^{n}$.

Define $\mathbf{E}^{n-2}(x)$ to be the orthogonal complement of $\mathbf{L}^{2}(x)$ in $\mathbf{L}_{x}^{n}$. Then $\mathbf{E}^{n-2}(x) \subseteq T_{0}(x)$. For, if $Y \in \mathbf{E}^{n-2}(x)$, then $\langle A Y, L\rangle=\langle Y, A L\rangle=0$ and, by (4.3)(iii), $\langle A Y, A L\rangle=\left\langle Y, A^{2} L\right\rangle=0$, so $A_{x}$ maps $\mathbf{E}^{n-2}(x)$ into itself. But the image of $A_{x}$ is $\operatorname{Span}\left\{A L_{x}\right\}$, so $A Y=0$ for all $Y \in \mathbf{E}^{n-2}(x)$.

Thus, $\mathbf{E}^{n-2}(x)$ and $A L_{x}$ play the role of $E$ and $\xi$ for $T_{0}(x)$ as described by (4.2). Note that $\mathbf{E}^{n-2}(x)$ depends on the choice of the local light field $L$. $L$ and $A L$ are differentiable in a neighborhood of $y$. It suffices then to show that $x \mapsto \mathbf{E}^{n-2}(x)$ defines a differentiable distribution near $y$.

Normalize $\{A L+L, A L-L\}$ at each $x$ near $y$ to give an orthonormal basis $\left\{T_{x}, S_{x}\right\}$ for $\mathbf{L}_{x}^{2}$, where $T$ and $S$ are differentiable unit time-like and space-like vector fields, respectively. Next, suppose $\mathbf{E}^{n-2}(y)=$ $\operatorname{Span}\left\{Y_{y}^{1}, \ldots, Y_{y}^{n-2}\right\}$. For $j=1, \ldots, n-2$, extend $Y_{y}^{j}$ to a vector field $Y^{j}$ in a neighborhood of $y$, and (in a possibly smaller neighborhood of $y$ ) define $Z^{j}$ by 


$$
Z^{j}=Y^{j}-\left\langle Y^{j}, S\right\rangle S+\left\langle Y^{j}, T\right\rangle T .
$$

Clearly, $\left\langle Z^{j}, S\right\rangle=\left\langle Z^{j}, T\right\rangle=0$, so $Z_{x}^{j} \in \mathbf{E}^{n-2}(x)$, for each $j$. Since $Z_{y}^{j}=Y_{y}^{j}$, $\left\{Z^{j}\right\}_{j=1}^{n-2}$ is linearly independent at $y$, and hence also at points near $y$. For those points $x, x \mapsto \mathbf{E}^{n-2}(x)$ is thus a differentiable distribution. Q.E.D.

Proposition (6.3). $T_{0}$ is integrable; so $T_{0} \mid(W \backslash G)$ foliates $W \backslash G$.

Proposition (6.4). The light lines in the relative nullity foliation are parallel along any $T_{0}$-direction.

Proof. If $Y \in T_{0}$, then it follows from the Codazzi equation (3.5) that $\nabla_{Y}(A L) y=-A([L, Y])_{y}$ lies in image $A_{y}=\operatorname{Span}\left\{A L_{y}\right\}$ if $y \in W \backslash G$. Q.E.D.

Proposition (6.5). $T_{0}$ is a totally geodesic foliation of $W \backslash G$.

Proof. The following conventions will be in order for a point $y \in W \backslash G$. The constructions are essentially those of the proof of Proposition (6.2). $L$ will be a local light field at $y$, so that $A L$ generates the light lines in the relative nullity spaces. $\mathbf{E}^{n-2}$ will denote the orthogonal complement of $\operatorname{Span}\{L, A L\}$ and have orthonormal basis $\left\{Z^{j}\right\}_{j=1}^{n-2}$. If $U \in \mathbf{L}_{y}^{n}$, then the following formula holds.

$$
U=\left(\sum_{j=1}^{n-2}\left\langle Z_{y}^{j}, U\right\rangle Z_{y}^{j}\right)+\frac{1}{\lambda(y)}\left(\left\langle U, L_{y}\right\rangle A L_{y}+\left\langle U, A L_{y}\right\rangle L_{y}\right)
$$

where $\lambda(y)=\left\langle A L_{y}, L_{y}\right\rangle \neq 0$.

Suppose $X$ and $Y$ are $T_{0}$ vector fields near $y \in W \backslash G$; then, $\langle Y, A L\rangle=0$ and

$$
\begin{aligned}
0 & =X \cdot\langle Y, A L\rangle=\left\langle\nabla_{X} Y, A L\right\rangle+\left\langle Y, \nabla_{X}(A L)\right\rangle \\
& =\left\langle\nabla_{X} Y, A L\right\rangle+\left\langle Y, \nabla_{L}(A X)\right\rangle+\langle Y, A([L, X])\rangle \\
& =\left\langle\nabla_{X} Y, A L\right\rangle+\langle A Y, A([L, X])\rangle=\left\langle\nabla_{X} Y, A L\right\rangle .
\end{aligned}
$$

By (6.6), this implies that the $L$-component of $\left(\nabla_{X} Y\right)_{y}=0$. That is, $\left(\nabla_{X} Y\right)_{y}$ $\in T_{0}(y)$. Q.E.D.

Proposition (6.5) implies that the leaf $M_{0}(y)$ of the relative nullity foliation through $y \in W \backslash G$ is an open piece of a hyperplane through $y$. This hyperplane carries a degenerate metric. Let $x_{0}$ be a fixed point of $W \backslash G$ and suppose $x_{t}$ is an affinely-parametrized geodesic in $M_{0}\left(x_{0}\right)$, where $0<t<b$. This geodesic can be extended to all values of the parameter in the complete space $L^{n}$. To show that $T_{0}$ furnishes a complete foliation of $W \backslash G$, we need, according to Lemma (5.9) (whose proof, except to show that $M_{0}\left(x_{0}\right) \subseteq G$, is independent of the specific metric), to demonstrate that $x_{b} \in W$. 
Suppose $\Omega$ generates the light line in $T_{0}\left(x_{0}\right)$. Extend $\Omega$ parallelly along $M_{0}\left(x_{0}\right)$. By Proposition (6.4), $\Omega_{y}$ generates the light line in $T_{0}(y)$ for each $y \in M_{0}\left(x_{0}\right)$. Now choose a light vector $L \in \mathbf{L}_{x_{0}}^{n}$ such that $\left\langle L, \Omega_{x_{0}}\right\rangle=-1$ and $A_{x_{0}} L \neq 0$. Then $A_{x_{0}} L=\rho_{0} \Omega_{x_{0}}$ with $\rho_{0} \neq 0$. Extend $L$ parallelly along $M_{0}\left(x_{0}\right)$; then $A L_{y}$ is a multiple of $\Omega_{y}$ for each $y \in M_{0}\left(x_{0}\right)$. Along $x_{t}$ for $t \in[0, b)$, $(A L)_{x_{t}}=\rho(t) \Omega_{t}$ where $\Omega_{t}=\Omega_{x_{t}}$ and $\rho(0)=\rho_{0}$. We can extend $L$ and $\Omega$ parallelly along $x_{t}$ for all $t$ as light vectors satisfying $\langle L, \Omega\rangle=-1$. If we define $\rho$ by $\rho(t)=-\langle A L, L\rangle_{x_{t}}$ for all $t$, then $(A L)_{x_{t}}=\rho(t) \Omega_{t}$ for $0<t<b$, but where $\rho(t)$ is a smoothly defined function for all $t$.

In what follows, when evaluating certain quantities at a fixed point $y=x_{t_{1}}$ on the geodesic, we will extend $\vec{x}_{t}$ and $L$, respectively, to a $T_{0}$-field $X$ and (e.g., by parallel extension in $\mathbf{L}^{n}$ ) a light field $L$, respectively, in a neighborhood $N_{y}$ of $y$.

At a point $y$ on the geodesic, we have, from the Codazzi equation,

$$
\nabla_{L}(A X)-A\left(\nabla_{L} X\right)=\nabla_{X}(A L)-A\left(\nabla_{X} L\right) \text {. }
$$

We wish to evaluate (6.7) at $y$. Since $X \in T_{0}, A X=0$ in $N_{y}$, and hence $\left(\nabla_{L}(A X)\right)_{y}=0$. Also, $L$ is parallel along $M_{0}\left(x_{0}\right)=M_{0}(y)$, so that $X_{y} \in T_{0}(y)$ implies that $A\left(\nabla_{X} L\right)_{y}=A(0)=0$. Thus, at $y,(6.7)$ becomes

$$
\left(\nabla_{X}(A L)\right)_{y}+A\left(\nabla_{L} X\right)_{y}=0 \text {. }
$$

Now, if $Z$ is a vector field, then $\nabla_{X} Z$ depends on the behavior of $Z$ on an integral curve of $X$ through the point at which $\nabla_{X} Z$ is evaluated. Here, the integral curve of $X$ through $y$ is the geodesic $x_{t}$, along which $(A L)_{x_{t}}=\rho(t) \Omega_{t}$ for $t \in[0, b)$. Since $\Omega_{t}$ is parallel along $x_{t}$, we have

$$
\left(\nabla_{X}(A L)\right)_{y}=\left(\nabla_{t}\left(\rho(t) \Omega_{t}\right)\right)_{t=t_{1}}=\rho^{\prime}\left(t_{1}\right) \Omega_{t_{1}} .
$$

Next, $\langle L, \Omega\rangle_{y}=-1$, so if $U \in \mathbf{L}_{y}^{n}$, then

$$
U=U^{\perp}-\left\langle U, L_{y}\right\rangle \Omega_{y}-\left\langle U, \Omega_{y}\right\rangle L_{y}
$$

where $U^{\perp} \in\left(\operatorname{Span}\left\{L_{y}, \Omega_{y}\right\}\right)^{\perp}$ (cf. (6.6)). Since $U^{\perp}, \Omega_{y} \in T_{0}(y)$,

$$
A U=-\left\langle U, \Omega_{y}\right\rangle A L_{y},
$$

and, in particular,

$$
A\left(\nabla_{L} X\right)_{y}=-\left\langle\left(\nabla_{L} X\right)_{y}, \Omega_{y}\right\rangle \rho\left(t_{1}\right) \Omega_{t_{1}} .
$$

Equations (6.8), (6.9) and (6.11) yield the following equation for $0<t<b$ :

$$
\left[\rho^{\prime}(t)-\rho(t)\left\langle\left(\nabla_{L} X\right)_{x_{t}}, \Omega_{t}\right\rangle\right] \Omega_{t}=0 .
$$

For $0 \leqslant t<b$, define $\psi(t)$ by

$$
\psi(t)=\left\langle\left(\nabla_{L} X\right)_{x_{t}}, \Omega_{t}\right\rangle
$$

Since $\rho$ is a well-defined smooth function, equation (6.12) implies that $\psi$ is a well-defined smooth function on $[0, b)$. The next step is to investigate the 
behavior of $\psi(t)$ along the geodesic $x_{t}$ for $t \in[0, b)$. First, extend $\Omega$ (which so far has been defined on $M_{0}\left(x_{0}\right)$ as well as along all of $x_{t}$ ) to a neighborhood of $y=x_{t}$ in $\mathbf{L}^{n}$ as follows. Define $\Omega$ by

$$
\Omega=\frac{-A L}{\langle A L, L\rangle}
$$

this extends $\Omega$ near $y$, and is well defined since $\langle A L, L\rangle$ is nonzero at, hence near, $y$. Points near $y$ lie in $W \backslash G$, so $\Omega$ generates the degenerate direction (or light line) of the relative nullity space at those points.

Now,

$$
\frac{d \psi}{d t}=X \cdot \psi=X \cdot\left\langle\nabla_{L} X, \Omega\right\rangle=\left\langle\nabla_{X} \nabla_{L} X, \Omega\right\rangle+\left\langle\nabla_{L} X, \nabla_{X} \Omega\right\rangle .
$$

Since $R \equiv 0$ and there is no torsion, equation (6.13) is equivalent to

$$
\frac{d \psi}{d t}=\left\langle\nabla_{L} \nabla_{X} X, \Omega\right\rangle-\left\langle\nabla_{\nabla_{L} X} X, \Omega\right\rangle+\left\langle\nabla_{\nabla_{X} L} X, \Omega\right\rangle+\left\langle\nabla_{L} X, \nabla_{X} \Omega\right\rangle .
$$

The last two terms vanish because $\nabla_{X} L=\nabla_{X} \Omega=0$ along $x_{t}$. Next, $\left(\nabla_{X} X\right)_{y}$ $=\left(\nabla, \vec{x}_{t}\right)_{t_{1}}=0$, so

$$
\left\langle\nabla_{L} \nabla_{X} X, \Omega\right\rangle_{y}=L_{y}\left\langle\nabla_{X} X, \Omega\right\rangle-\left\langle\left(\nabla_{X} X\right)_{y},\left(\nabla_{L} \Omega\right)_{y}\right\rangle=L_{y}\left\langle\nabla_{X} X, \Omega\right\rangle .
$$

Since $T_{0}$ is totally geodesic, $\nabla_{X} X \in T_{0}$. Therefore, $\left\langle\nabla_{X} X, \Omega\right\rangle$, and hence $L_{y}\left\langle\nabla_{X} X, \Omega\right\rangle$, vanishes. Finally, consider the algebraic decomposition

$$
\left(\nabla_{L} X\right)_{y}=\left(\nabla_{L} X\right)_{0}-\left\langle\left(\nabla_{L} X\right)_{y}, \Omega_{y}\right\rangle L_{y}
$$

where $\left(\nabla_{L} X\right)_{0} \in T_{0}(y)$ (cf. (6.6)). $T_{0}$ is totally geodesic and $\Omega_{y}$ generates the degenerate direction of $T_{0}(y)$, so $\left\langle\nabla_{\left(\nabla_{L} X\right)_{0}} X, \Omega_{y}\right\rangle=0$. Therefore,

$$
\begin{aligned}
\left\langle\nabla_{\nabla_{L} X} X, \Omega\right\rangle_{y} & =-\left\langle\nabla_{\left.\left\langle\left(\nabla_{L} X\right)_{y}, \Omega_{y}\right\rangle L_{y} X, \Omega\right\rangle_{y}}\right. \\
& =-\left\langle\left(\nabla_{L} X\right)_{y}, \Omega_{y}\right\rangle\left\langle\nabla_{L} X, \Omega\right\rangle_{y}=-\psi\left(t_{1}\right)^{2} .
\end{aligned}
$$

Thus, (6.14) becomes

$$
\frac{d \psi}{d t}=\psi(t)^{2}
$$

for $0 \leqslant t<b$.

If $\psi(a)=0$ for some $a \in[0, b)$, then $\psi \equiv 0$ or $[0, b)$. Otherwise, it is easily seen that

$$
\psi(t)=\frac{\psi(0)}{1-\psi(0) t}
$$

for $0 \leqslant t<b$.

Now, to show that $x_{b} \in W$ is to show that $(A L)_{x_{b}} \neq 0$. But

$$
(A L)_{x_{b}}=\lim _{t \rightarrow b}(A L)_{x_{t}}=\lim _{t \rightarrow b} \rho(t) \Omega_{t}=\left(\lim _{t \rightarrow b} \rho(t)\right) \Omega_{b} .
$$


Recall that $\rho$ is a well-defined smooth function for all $t$, so $\lim _{t \rightarrow b} \rho(t)$ exists and equals $\rho(b)$. Equation (6.12) implies

$$
\rho(t)=\rho_{0} \exp \left(\int_{0}^{t} \psi(s) d s\right), \quad 0 \leqslant t<b .
$$

If $\psi \equiv 0$, then $\rho(t)=\rho_{0}$, so $(A L)_{x_{b}}=\rho_{0} \Omega_{b} \neq 0$. If (6.17) holds, then (6.18) implies that

$$
\rho(t)=\frac{\rho_{0}}{|1-\psi(0) \cdot t|}, \quad 0 \leqslant t<b .
$$

Because $\rho$ is smoothly defined for all $t, b \neq 1 / \psi(0)$ and $\rho(b)=\rho_{0} / \mid 1-$ $\psi(0) b \mid \neq 0$, implying that $x_{b} \in W$.

As in the proof of Theorem (5.14), that $x_{b} \in W$ implies that

$$
\sup \left\{u: x_{t} \in M_{0}\left(x_{0}\right) \text { for } 0 \leqslant t<u\right\}
$$

is infinite, and gives the following results.

Theorem (6.19). $T_{0}$ is a complete totally geodesic foliation of $W \backslash G$.

COROLlARY (6.20). For each $x \in W \backslash G, M_{0}(x)$ is a hyperplane contained in $W \backslash G$.

COROLlaRY (6.21). $W \backslash G$ is a union of parallel hyperplanes through points of an open set.

Since $G$ and $W \backslash G$ are disjoint open sets comprising $W$, Theorems (5.14) and (6.19) yield the following pivotal result.

THEOREM (6.22). $T_{0}$ is a complete totally geodesic foliation of $W$.

In a similar manner, their corollaries combine to give this description of the umbilic-free set $W$.

THEOREM (6.23). The open set $W$ is the union of a family of parallel hyperplanes.

Of course, each such hyperplane is a leaf of the relative nullity foliation, and parallel hyperplanes must have the same inherited metric:

THEOREM (6.24). Either $G=\varnothing$ or $G=W$.

\section{PART III. THE ClASSIFICATION THEOREMS}

7. Preliminary remarks. In this part of the paper, we classify, up to a proper motion of $\mathbf{L}^{n+1}$, all isometric immersions: $\mathbf{L}^{n} \rightarrow \mathbf{L}^{n+1}$. To do this, we prove an appropriate "Moore's Lemma", use the results of Part II to divide our discussion into two cases $(G=\varnothing$ and $G=W)$, and then, for each case, describe the structure induced on $L^{n}$ by $T_{0}$. To obtain our desired description 
of the immersion $f: \mathbf{L}^{n} \rightarrow \mathbf{L}^{n+1}$, it then remains to see how the structure induced by $T_{0}$ is mapped by $f$ into $L^{n+1}$. To the degenerate case $(G=\varnothing)$, the results of Part I will be pertinent.

For the remainder of this section, let $M=M_{0} \times M_{1}$ be a product of connected manifolds-with-connection. Hence, if $X$ is a tangent vector field on one factor and $Y$ is a tangent vector field on the other, then $\nabla_{X} Y=0$. Suppose further that $M$ has a nondegenerate metric compatible with its connection. Let $f: M \rightarrow \mathbf{R}^{N}$ be an isometric immersion of $M$ into a real vector space with nondegenerate metric and the usual flat connection. We assume there is a point $\left(x_{0}, y_{0}\right) \in M$ with $f\left(x_{0}, y_{0}\right)=0 \in \mathbf{R}^{N}$. Define $f_{i}: M_{i} \rightarrow \mathbf{R}^{N}$ $(i=0,1)$ as follows:

$$
\begin{aligned}
& \text { if } x \in M_{0}, f_{0}(x)=f\left(x, y_{0}\right) ; \\
& \text { if } y \in M_{1}, f_{1}(y)=f\left(x_{0}, y\right) .
\end{aligned}
$$

If $X, Y$ are tangent vector fields on $M$, then let

$$
D_{X} f_{*} Y=f_{*}\left(\nabla_{X} Y\right)+\alpha(X, Y)
$$

describe the decomposition of $D_{X} f_{*} Y$ into tangential and normal components. (Here, $D$ is the covariant differentiation with respect to the flat connection on $\mathbf{R}^{N} ; \nabla$ is the covariant differentiation on $M$.)

LemMa (7.1) ("MOORE's LEMMA" [M]). If, everywhere on $M, \alpha(X, Y)=0$ whenever $X$ is tangent to $M_{0}$ and $Y$ is tangent to $M_{1}$, then $f(x, y)=f_{0}(x)+$ $f_{1}(y)$ for every $(x, y) \in M$.

Proof. First, notice that if $X$ is tangent to $M_{0}$ and $Y$ is tangent to $M_{1}$, the hypothesis implies that $D_{X} f_{*} Y=0$. Therefore,

(7.2) If $y \in M_{1}, Y \in T_{y} M_{1}$, and $x_{t}$ is a curve in $M$, then the vector field $f_{*(x, y)}(0, Y)$ along $f\left(x_{t}, y\right)$ is parallel in $\mathbf{R}^{N}$.

Let $x_{t}$ be a curve from $x_{0}$ to $x$ in $M_{0}$ and let $y_{t}$ be a curve from $y_{0}$ to $y$ in $M_{1}$. Consider the curves $f\left(x, y_{t}\right)$ and $f_{0}(x)+f_{1}\left(y_{t}\right)$ in $\mathbf{R}^{N}$. If $t=0$, they both take the value $f\left(x, y_{0}\right)$. On the other hand,

$$
\frac{d}{d t}\left[f_{0}(x)+f_{1}\left(y_{t}\right)\right]=\frac{d}{d t} f\left(x_{0}, y_{t}\right)=f_{*\left(x_{0} y_{t}\right)}\left(0, \vec{y}_{t}\right)
$$

and

$$
\frac{d}{d t} f\left(x, y_{t}\right)=f_{*(x, y)}\left(0, \vec{y}_{t}\right) .
$$

By (7.2) the two curves have the same tangent vectors. They agree at $t=0$ and hence coincide. Q.E.D.

8. The nondegenerate case $(G=W)$. Fix an origin 0 of $\mathbf{L}^{n}$ in $W$, and let $M_{0}$ be the hyperplane-leaf through 0 . For a unit vector $Z$ in $T_{0}^{\perp}(0)$, denote $\operatorname{Span}\{Z\}$ by $t Z(t \in \mathbf{R})$. Then 


$$
\mathbf{L}^{n}=M_{0} \times t Z=\left\{(x, t): x \in M_{0}, t \in \mathbf{R}\right\}
$$

describes $\mathbf{L}^{n}$ both as a direct product of vector spaces and a direct product of pseudo-Riemannian manifolds. If $z=(x, t) \in W$, then $M_{0}(z)=\{(y, t): y \in$ $\left.M_{0}\right\}$, since the leaves of $T_{0}$ are parallel hyperplanes. If $z=(x, t) \notin W$, then define $M_{0}(z)=\left\{(y, t): y \in M_{0}\right\}$ and $T_{0}(z)=T_{z}\left(M_{0}(z)\right)$. In either case, $\partial / \partial t$ generates the orthogonal complement of $T_{0}(z)$.

We may make the following assertions for all $z \in \mathbf{L}^{n}$. If $X \in T_{0}(z)$, then $A X=0 ; A(\partial / \partial t)=\lambda(z) \partial / \partial t$, with $\lambda(z) \neq 0$ just in case that $z \in W$. The Codazzi equation (3.5) gives, in analogy with (5.6), $(X \cdot \lambda)(\partial / \partial t)+\lambda\left(\nabla_{t} X\right)^{\perp}$ $=0$. Since the $T_{0}$ planes are, or are chosen to be, parallel, $\left(\nabla_{t} X\right)^{\perp}=0$. Therefore, $X \cdot \lambda=0$.

Proposition (8.2). $\lambda$ is constant on each $M_{0}(z)$; hence, $\lambda$ is a function of $t$.

Now consider the immersion $f: \mathbf{L}^{n} \rightarrow \mathbf{L}^{n+1}$. Assume $f(0)=0$. Take $M_{0}$ as in (8.1), and define $M_{1}=t Z . M=\mathbf{L}^{n}$ and $\mathbf{R}^{N}=\mathbf{L}^{n+1}$ clearly have nondegenerate metrics. If $X \in T_{0}(z)=T_{z}\left(M_{0}\right)$ and $Y \in T_{z} M_{1}$ at $z \in L^{n}$, then $\alpha(X, Y)=h(X, Y) \xi=\langle A X, Y\rangle \xi=\langle 0, Y\rangle \xi=0$, where $\xi$ is a unit normal field on $f\left(\mathbf{L}^{n}\right)$. Therefore, writing $f_{1}(t)=f_{1}(t Z)$, we have according to "Moore's Lemma"

$$
f(x, t)=f_{0}(x)+f_{1}(t), \quad(x, t) \in M_{0} \times \mathbf{R} .
$$

Proposition (8.4). $f_{0}=f \mid M_{0}$ is an isometry of $M_{0}$ onto an $(n-1)$-plane in $\mathbf{L}^{n+1}$.

Proof. Let $x_{s}=\left(x_{s}, 0\right)$ be a geodesic line emanating from 0 in the hyperplane $M_{0}$. (So $x_{s}=s \vec{x}_{0}$.) Then

$$
D_{s} \overrightarrow{f\left(x_{s}, 0\right)}=D_{s} f_{*}\left(\vec{x}_{s}, 0\right)=f_{*}\left(\nabla_{s} \vec{x}_{s}\right)+\left\langle A \vec{x}_{s}, \vec{x}_{s}\right\rangle \xi=0 \text {. }
$$

Thus $f\left(s \vec{x}_{0}\right)=s f_{*}\left(\vec{x}_{0}, 0\right)$, and $f\left(M_{0}\right)$ is an $(n-1)$-plane spanned by $f_{* 0} T_{0}(M)$. Q.E.D.

Corollary (8.5). If $z=(x, t) \in \mathbf{L}^{n}, f\left(M_{0}(z)\right)=f_{0}\left(M_{0}\right)+f_{1}(t)$. Hence, $\left\{f\left(M_{0}(z)\right): z \in \mathbf{L}^{n}\right\}$ is a family of parallel $(n-1)$-planes in $\mathbf{L}^{n+1}$.

For $z \in \mathbf{L}^{n}$, let $\left[f\left(M_{0}(z)\right)\right]^{\perp}$ be the plane in $\mathbf{L}^{n+1}$ at $f(z)$ orthogonal to $f\left(M_{0}(z)\right) . f$ is an isometry, so the planes $\left[f\left(M_{0}(z)\right)\right]^{\perp}$ are parallel, and $f_{1}(t)$ lies in the plane $\left[f\left(M_{0}\right)\right]^{\perp}$. Writing $\mathbf{L}^{n+1}$ as the direct product $f\left(M_{0}\right) \times\left[f\left(M_{0}\right)\right]^{\perp}$, we may now write

$$
f(x, t)=\left(f_{0}(x), f_{1}(t)\right) \in f\left(M_{0}\right) \times\left[f\left(M_{0}\right)\right]^{\perp}
$$

for $(x, t) \in M_{0} \times \mathbf{R}=\mathbf{L}^{n}$. Since the relative nullities are nondegenerate, there are, by Theorem (1.1), only two cases to consider. 
(i) $M_{0} \approx \mathbf{E}^{n-1}$. Then $f\left(M_{0}\right)^{\perp}$ is a Lorentz plane $\mathbf{L}^{2}$ in which $f_{1}(t)$ is a unit speed time-like curve $c(\langle d c / d t, d c / d t\rangle=-1)$. Since $f_{0}\left(s \vec{x}_{0}\right)=s f_{* 0}\left(\vec{x}_{0}, 0\right)$ for $\vec{x}_{0} \in T_{0}(0)=M_{0}$, we may treat $f_{0}$ as the identity map of $\mathbf{E}^{n-1}$ onto itself (up to a proper motion of $\mathbf{L}^{n+1}$ ).

(ii) $M_{0} \approx \mathbf{L}^{n-1}$. Then $f\left(M_{0}\right)^{\perp}$ is a Euclidean plane $\mathbf{E}^{2}$ in which $f_{1}(t)$ is a unit-speed curve $c(\langle d c / d t, d c / d t\rangle=1)$. In analogy with (i), we may treat $f_{0}$ as the identity map of $\mathbf{L}^{n-1}$ onto itself (up to a proper motion of $\mathbf{L}^{n+1}$ ).

(8.7). FirST CLASSIFICATION THEOREM. Up to a proper motion of $\mathbf{L}^{n+1}$, the isometric immersions: $\mathbf{L}^{n} \rightarrow \mathbf{L}^{n+1}$ with nondegenerate relative nullities have one of the following forms:

(i) $\mathbf{E}^{n-1} \times \mathbf{L}^{1} \rightarrow^{\text {id } \times c} \mathbf{E}^{n-1} \times \mathbf{L}^{2}$, with c a unit-speed time-like curve in $\mathbf{L}^{2}$,

(ii) $\mathbf{L}^{n-1} \times \mathbf{E}^{1} \rightarrow^{\mathrm{id} \times c} \mathbf{L}^{n-1} \times \mathbf{E}^{2}$, with $c$ a unit-speed Euclidean plane curve. (All products are orthogonal.)

The surfaces described by Theorem (8.7) are cylinders over plane curves as mentioned in the example (3.8) and as in the Hartman-Nirenberg theorem (see (0.1)). Indeed the proof of (8.7) also shows that all isometric immersions: $\mathbf{E}^{n} \rightarrow \mathbf{L}^{n+1}$ have the form

$$
\text { id } \times c: \mathbf{E}^{n-1} \times \mathbf{E}^{1} \rightarrow \mathbf{E}^{n-1} \times \mathbf{L}^{2}
$$

where $c$ is a unit-speed space-like curve in $\mathbf{L}^{2}$.

Furthermore, there is a relationship between the nonzero eigenvalue of the second fundamental tensor and the curvature of the plane curve $c$ in each of those theorems, which we will briefly outline in the case of Theorem (8.7).

In case (ii) of that theorem, at each point $z \in \mathbf{L}^{n},\left[f\left(M_{0}(z)\right)\right]^{\perp}$ is spanned orthonormally by $f_{* z} \partial / \partial t=d / d t\left(f_{1}(t)\right)$ and $\xi_{z}$ (where $\xi$ is a local unit normal field). Now, if $z=(x, t)$, one may use (3.1), (3.2) and (8.2) to show

$$
D_{t} f_{* z}\left(\frac{\partial}{\partial t}\right)=\lambda(t) \xi_{z}, \quad D_{t} \xi_{z}=-\lambda(t) f_{*}\left(\frac{\partial}{\partial t}\right) .
$$

Thus, for each $t_{1} \in \mathbf{R}, \lambda(t)$ is the curvature of $c=f_{1}(t)$ at $t=t_{1}$.

If $c: \mathbf{L}^{1} \rightarrow \mathbf{L}^{2}$ is a unit-speed time-like curve in $\mathbf{L}^{2}$, then let $e_{1}=d c / d t$ be the unit tangent vector, and let $e_{2}$ be the unit (space-like) normal to the curve. Then $\left(e_{1}, e_{2}\right) \in \mathrm{O}(1,1)$. Since $\left\langle d e_{i} / d t, e_{i}\right\rangle=0(i=1,2),(1.3)$ and (1.5) imply the equations

$$
\frac{d e_{1}}{d t}=-k(t) e_{2}, \quad \frac{d e_{2}}{d t}=-k(t) e_{1} .
$$

In analogy with the case of Euclidean plane curves, $k$ could be called the curvature of the curve $c$.

Now in case (i) of (8.7), at each point $z \in \mathbf{L}^{n},\left(f\left(M_{0}(z)\right)\right)^{\perp}$ is spanned orthonormally by the time-like $f_{* z}(\partial / \partial t)=d f_{1}(t) / d t$ and the space-like unit normal $\xi_{z}$. If $z=(x, t)$, one may in this case derive 


$$
D_{t} f_{*}\left(\frac{\partial}{\partial t}\right)=-\lambda(t) \xi_{z}, \quad D_{t} \xi=-\lambda(t) f_{*}\left(\frac{\partial}{\partial t}\right)
$$

Since the equations (8.9) fit the form (8.8), $\lambda\left(t_{1}\right)$ can be considered as the curvature of $c=f_{1}(t)$ at $t=t_{1}$.

9. The degenerate case $(G=\varnothing)$. Choose the origin 0 of $\mathbf{L}^{n}$ to be in $W$, and let $M_{0}$ be the hyperplane-leaf through $0 . M_{0}$ is a degenerate hyperplane, so there is a unique light line contained in $M_{0}$, generated by $\Omega$. Now let $L$ be a tangent light vector at 0 such that $\langle L, \Omega\rangle=-1$ and $A L \neq 0$; then $A L=\rho \Omega$. Denote $\operatorname{Span}\{L\}$ by $s L(s \in \mathbf{R})$ and $\operatorname{Span}\{\Omega\}$ by $u \Omega(u \in \mathbf{R})$. Then

$$
\mathbf{L}^{n}=M_{0} \oplus s L
$$

describes $\mathbf{L}^{n}$ as a direct sum of vector spaces.

Let $E$ be the $(n-2)$-dimensional subspace in $M_{0}$ which is the orthogonal complement in $\mathbf{L}^{n}$ of the Lorentz plane $\operatorname{Span}\{L, \Omega\}$. Then

$$
\mathbf{L}^{n}=E \times \operatorname{Span}\{L, \Omega\}
$$

is a decomposition of $\mathbf{L}^{n}$ both as an orthogonal sum of vector spaces and as a product of pseudo-Riemannian manifolds. We also have a direct sum

$$
\mathbf{L}^{n}=E \oplus u \Omega \oplus s L \text {. }
$$

Representing a point of $\mathbf{L}^{n}$ by $(p, u, s)(p \in E ; u, s \in \mathbf{R})$ provides a global flat chart on $\mathbf{L}^{n}$.

If $z=(p, u, s) \in W$, then $M_{0}(z)=\{(q, v, x): q \in E, v \in \mathbf{R}\}$ since both sets are hyperplanes parallel to $M_{0}$. If $z=(p, u, s) \notin W$, then define $M_{0}(z)$ to be as above, with $T_{0}(z)=T_{z}\left(M_{0}(z)\right)$. Each $M_{0}(z)$ contains a unique light line, $\{(p, v, s): v \in \mathbf{R}\}$, spanned by $(\partial / \partial u)_{z}$. For each $z \neq 0$ in $\mathbf{L}^{n}$, define

$$
E(z)=\{(q, u, s): q \in E\} \text {. }
$$

Thus, $M_{0}$ and its decomposition $M_{0}=E \oplus \operatorname{Span}\{\partial / \partial u\}$ are parallel throughout $\mathbf{L}^{n}$.

If $z$ is any point in $\mathbf{L}^{n}$, we may say the following: $(\partial / \partial s)_{z}$ is a tangent light vector at $z$ with $\langle\partial / \partial s, \partial / \partial u\rangle_{z}=-1$. Also $(A \cdot \partial / \partial s)_{z}=\rho(z)(\partial / \partial u)_{z}$ with $\rho(z) \neq 0$ just in case $z \in W$.

Proposition (9.2). $\rho$ is a constant on each $M(z), z \in \mathbf{L}^{n}$. Hence, $\rho$ is a function of $s$.

Proof. Let $z \in W$ (otherwise $\rho \equiv 0$ on $M_{0}(z)$ ), and let $x_{t}$ be any geodesic in $M_{0}(z)$ emanating from $z$. For each $t$, write $\rho(t)$ for $\rho\left(x_{t}\right)$, and $\Omega_{t}$ for $(\partial / \partial u)_{x_{i}}$. Then $(A \partial / \partial s)_{x_{t}}=\rho(t) \Omega_{t}$. Applying the derivation of (6.12) in this case gives

$$
\frac{d \rho}{d t}-\left\langle\nabla_{s} \vec{x}_{t}, \Omega_{t}\right\rangle \rho(t)=0
$$


and, in particular, that $\left\langle\nabla_{s} \vec{x}_{t}, \Omega_{t}\right\rangle$ is independent of the extension of $\vec{x}_{t}$ to a $T_{0}$-field near $x_{t}$. But $\nabla_{s} \vec{x}_{t} \in T_{0}$ because the $M_{0}$ 's are parallel hyperplanes in $\mathbf{L}^{n}$. Since $\Omega_{t}$ generates the degenerate line in $T_{0}\left(x_{t}\right),\left\langle\nabla_{s} \vec{x}_{t}, \Omega_{t}\right\rangle=0$. This implies that $\rho(t)=\rho(z)$ for all $t$. Q.E.D.

We now turn our attention to the immersion $f: \mathbf{L}^{n} \rightarrow \mathbf{L}^{n+1}$ under consideration. Assume that $f(0)=0$. Take $M_{0}$ as in (9.1) and define $M_{1}=$ $s L$. Then $M=\mathbf{L}^{n}=M_{0} \times M_{1}$ is a direct, though not orthogonal, product of manifolds-with-connection. $M$ has a nondegenerate metric, as does $\mathbf{R}^{N}=$ $\mathrm{L}^{n+1}$. If $X \in T_{0}(z)=T_{z} M_{0}$ and $Y \in T_{z} M_{1}=\operatorname{Span}\left\{(\partial / \partial s)_{z}\right\}$, then $\alpha(X, Y)$ $=\langle A X, Y\rangle \xi=0$, where $\xi$ is a local unit normal field. "Moore's Lemma" allows us to write $f(p, u, s)=f_{0}(p, u)+f_{1}(s)$ for $(p, u, s) \in E \times u \Omega \times s L$ $=\mathbf{L}^{n}$.

If $x_{t}$ is a geodesic line in $M_{0}(z)$ emanating from $z$ (so that $x_{t}=z+t \vec{x}_{0}$ ) then, as with (8.4), $f\left(x_{t}\right)=z+t f_{* z}\left(\vec{x}_{0}\right)$.

From this follow the next three facts.

Proposition (9.3). $f$ is an isometry of $M_{0}(z)$ onto a (degenerate) $(n-1)$ plane in $\mathbf{L}^{n+1}$.

Proposition (9.4). $f$ is an isometry of $E(z)$ onto a Euclidean $(n-2)$-plane in the $(n-1)$-plane $f\left(M_{0}(z)\right)$.

Proposition (9.5). $f$ maps the light line in $M_{0}(z)$ onto the light line in the $(n-1)$-plane $f\left(M_{0}(z)\right)$.

Proposition (9.6). Each of $\left\{f\left(M_{0}(z)\right)\right\}_{z \in \mathbf{L}^{n}}$ and $\{f(E(z))\}_{z \in \mathbf{L}^{n}}$ is a parallel family.

Proof. If $X$ is a vector field in either $T_{0}$ or $E$, and $Y$ is any vector field on $\mathbf{L}^{n}$, then

$$
D_{Y} f_{*} X=f_{*}\left(\nabla_{Y} X\right)+\langle Y, A X\rangle \xi=f_{*}\left(\nabla_{Y} X\right) \in f_{*} T_{0} \text { or } \in f_{*} E \text {. Q.E.D. }
$$

Before considering the general immersion $\mathbf{L}^{n} \rightarrow \mathbf{L}^{n+1}$ (with degenerate relative nullity), we examine the case $n=2$, where $E=\varnothing$ and $T_{0}(0)=$ $\operatorname{Span}\{\Omega\}$, so $\mathbf{L}^{2}=u \Omega \times s L$. If $z \in \mathbf{L}^{2}$, then $\mathbf{L}^{3}$ is spanned by the null frame $\left(f_{* z}(\partial / \partial s), f_{* z}(\partial / \partial u), \xi_{f(z)}\right)$ where $\xi$ is a local unit (space-like) normal field. It will be evident that we may assume that this frame is proper, without any loss of generality.

Now:

(i) $d f_{1} / d s=f_{*}(\partial / \partial s)$,

(ii) $D_{s} f_{*}(\partial / \partial s)=f_{*}\left(\nabla_{s} \partial / \partial s\right)+\langle A \partial / \partial s, \partial / \partial s\rangle \xi=-\rho(s) \xi$,

(iii) $D_{s} f_{*} \partial / \partial u=\rho(s)\langle\Omega, \Omega\rangle \xi=0$,

(iv) $D_{s} \xi=-f_{*}(A \partial / \partial s)=-\rho(s) f_{*}(\partial / \partial u)$.

Statements (i)-(iv) say that $\left(f_{1}(s), F(s)\right)$, where $F(s)$ is the frame 
$\left(f_{*} \partial / \partial s, f_{*} \partial / \partial u, \xi\right)$, is a framed null curve that in fact is a GNC (cf. equations (3.11)). Since (7.2) and (9.5) imply that the immersion $f$ is given by

$$
f(u, s)=f_{0}(u)+f_{1}(s)=f_{1}(s)+u f_{*}(\partial / \partial u),
$$

the immersed image of $\mathbf{L}^{2}$ under $f$ is the $B$-scroll of that GNC. We shall call $f$ a " $B$-scroll immersion" of $\mathbf{L}^{2}$ into $\mathbf{L}^{3}$.

(9.7). SECOND CLASSIFICATION THEOREM: SURFACE VERSION. The isometric immersions $\mathbf{L}^{2} \rightarrow \mathbf{L}^{3}$ with degenerate relative nullities are precisely the $B$-scroll immersions.

If $n>2$, then the Lorentz 3-spaces orthogonal to $f(E(z))$ in $\mathbf{L}_{f(z)}^{n+1}$ (for $z \in \mathbf{L}^{n}$ ) are parallel in $\mathbf{L}^{n+1}$, by Proposition (9.6). If $X$ is an $E$-vector field, then $\left\langle f_{*} X, f_{*} \partial / \partial s\right\rangle=\langle X, \partial / \partial s\rangle=0$, so $f_{1}(s)$ lies in the Lorentz 3-space orthogonal to $f(E)$. Also, $\left\langle f_{*} X, f_{*} \partial / \partial u\right\rangle=0$. Now write

$$
\mathbf{L}^{n}=\mathbf{E}^{n-2} \times \mathbf{L}^{2}=E \times \operatorname{Span}\{\Omega, L\}
$$

and write

$$
\mathbf{L}^{n+1}=\mathbf{E}^{n-2} \times \mathbf{L}^{3}=f(E) \times \operatorname{Span}\left\{f_{*} \Omega, f_{*} L, \xi_{0}\right\}
$$

where $\xi_{0}$ is a unit normal at $0 \in \mathbf{L}^{n+1}$. Then $f$ factors

$$
\left.f\right|_{E} \times\left. f\right|_{\mathbf{L}^{2}}: \mathbf{E}^{n-2} \times \mathbf{L}^{2} \rightarrow \mathbf{E}^{n-2} \times \mathbf{L}^{3} .
$$

By Proposition (9.4), we may assume that, up to a proper Lorentz motion of $\mathbf{L}^{n+1},\left.f\right|_{E}$ is the identity map of $\mathbf{E}^{n-2}$ onto itself. Proposition (9.7) applies to $\left.f\right|_{\mathbf{L}^{2}}$.

(9.8). SECOND CLASSIFICATION THEOREM: MAIN VERSION. Up to a proper motion of $\mathbf{L}^{n+1}$, the isometric immersions: $\mathbf{L}^{n} \rightarrow \mathbf{L}^{n+1}$ with degenerate relative nullities have the form

$$
\mathbf{E}^{n-2} \times \mathbf{L}^{2} \stackrel{\text { id } \times g}{\rightarrow} \mathbf{E}^{n-2} \times \mathbf{L}^{3}
$$

where the factors in each product are orthogonal, and the map $g: \mathbf{L}^{2} \rightarrow \mathbf{L}^{3}$ is a $B$-scroll immersion.

We remark that the hypersurfaces described by (8.7) and the HartmanNirenberg theorem are "built" over plane curves, whereas those described by (9.7) and (9.8) are "built" over certain curves in a 3-dimensional space. That space curves rather than plane curves are required there is the import of the following observation, whose straightforward proof is omitted.

Proposition (9.9). Suppose $\mathbf{L}^{2}=\operatorname{Span}\{\xi, Y\}$ with $\xi$ a light vector and $\langle\xi, Y\rangle=k$, a constant. Suppose $\eta$ is a light vector in $\mathbf{L}^{3}$ such that

$$
f(u, v)=f(u \xi+v Y)=x(v)+u \eta
$$


is an isometric immersion: $\mathbf{L}^{2} \rightarrow \mathbf{L}^{3}$. If $x(v)$ is a plane curve, then $x(v)$ is a line, and hence the immersion is totally geodesic.

\section{REFERENCES}

[A] K. Abe, Characterization of totally geodesic submanifolds in $S^{n}$ and $C P^{n}$ by an inequality, Tôhoku Math. J. 23 (1971), 219-244.

[B] W. Bonnor, Null curves in a Minkowski space-time, Tensor (N.S.) 20 (1969), 229-242.

[F] D. Ferus, On the completeness of nullity foliations, Michigan Math. J. 18 (1971), 61-64.

[G] W. Greub, Linear algebra, 2nd ed., Springer-Verlag, New York, 1963.

[HN] P. Hartman and L. Nirenberg, On spherical image maps whose Jacobians do not change sign, Amer. J. Math. 81 (1959), 901-920.

[KN] S. Kobayashi and K. Nomizu, Foundations of differential geometry. I, II, Interscience, New York, 1963, 1969.

[M] J. D. Moore, Isometric immersions of Riemannian products, J. Differential Geometry 5 (1971), 159-168.

$\left[\mathbf{N}_{1}\right] \mathrm{K}$. Nomizu, On hypersurfaces satisfying a certain condition on the curvature tensor, Tôhoku Math. J. 20 (1968), 46-59.

$\left[\mathrm{N}_{2}\right]$, Lectures on differential geometry of submanifolds (unpublished lecture notes), Brown University, 1975.

[S] M. Spivak, A comprehensive introduction to differential geometry. II, III, Publish or Perish, Boston, Mass., 1970, 1975.

Department of Mathematics, Brown University, Providence, Rhode Island 02912

Daniel H. Wagner, Associates, Station Square One, Paol, Pennsylvania 19301 (Current address) 